\title{
Constraint Satisfied Model Predictive Control Strategy for MMC Energy Storage System Based on Super Capacitor
}

\author{
Shi Yin ${ }^{1 *}$, Guanjun $\mathrm{Li}^{1}$, Zhong Liu ${ }^{2}$, Yibin Tao \\ ${ }^{1}$ National Key Laboratory on Operation and Control of Renewable Energy and Energy Storage, China Electric Power Research Institute \\ Co., Ltd, Nanjing, Jiangsu, 210003, China \\ ${ }^{2}$ Yangzhou Power Supply Company, State Grid Jiangsu Electric Power Co., Ltd, Yangzhou, Jiangsu, 225000, China
}

\begin{abstract}
With the continuous development of power electronics technology and the large-scale access of new energy power generation, the stable operation of the power grid is facing huge challenges. The MMC energy storage system has attracted more and more attention due to its strong ability to support the grid. However, the MMC energy storage system has a complex structure and contains many devices, and the research on high-performance control technology has always been a difficult point. In response to the above problems, this article proposes a constraint satisfaction model predictive control method for MMC energy storage system based on super capacitor. In the article, the operation mechanism of MMC energy storage system is analyzed, and the discrete domain mathematical model of MMC-ESSC is established. The article studies the prediction method of the future internal and external variables of the system, the rolling optimization mechanism and the method of establishing the objective function, and finally carries on the experiments verification. The analysis of experimental results shows that proposed control technology has high dynamic characteristics and efficiency.
\end{abstract}

\section{Introduction}

In recent years, with the rapid development of power electronic technology and the improvement of control theory, higher control requirements have been put forward for power electronic systems[1]. The modular multi-level energy storage system based on supercapacitors has the modular structure design, common DC bus structure, high output quality, and high dynamic response rate. It has received extensive attention in flexible DC transmission and DC distribution networks[2]. It has gradually become an ideal form of medium voltage energy storage system with DC grid voltage support and power supply for important loads[3]. The modular multi-level energy storage system based on supercapacitors has a modular structure design, a common DC bus structure, high output quality, and high dynamic response rate. It has received extensive attention in flexible DC transmission and DC distribution networks. It has gradually become an ideal form of medium voltage energy storage system with DC grid voltage support and power supply for important loads[45].

Multiple types of constraint indicators and control targets have been added to the design of the electric power electronics system. In view of such numerous constraints and control targets, the traditional linear PI control has limitations in dealing with the constraints of multi-target optimization problems[6]. At this time, the potential advantage of model predictive control (MPC) to solve complex and miscellaneous constraints and optimization problems is obvious.

In order to solve the above problems, this paper has proposed a constraint satisfied model predictive control strategy for MMC-ESSC. This control strategy does not require control links such as rotating coordinate system transformation, inner and outer loop control coordination, PWM modulation module and additional sub-module voltage equalization. By predicting the future state of the system, establishing an appropriate rolling optimization mechanism, and introducing a weighting factor to reduce system loss while meeting the system output quality, the proposed control method can improve the dynamic response capability of the system and realize the integration of different control objectives.

\section{Operation mechanism of MMC-ESSC}

The MMC-ESSC system is based on a modular multilevel topological structure, which is a three-phase six-arm symmetrical structure. Each bridge arm is composed of $\mathrm{N}$ sub-modules and bridge arm inductors in series. The sub-module is a half-bridge converter structure, and the DC side uses a super capacitor as an energy storage unit.

yinshi@epri.sgcc.com.cn 
The simplified circuit of MMC-ESSC is shown in Figure 1. The synthesis of the upper and lower bridge arm voltages $\left(v_{j k} j=\mathrm{a}, \mathrm{b}, \mathrm{c}\right.$ and $\left.k=\mathrm{p}, \mathrm{n}\right)$ are through the switching of the control sub-modules. In order to achieve the stability of the DC voltage, the total input of the upper and lower bridge arm sub-modules must be kept at $\mathrm{N}$ in real time.

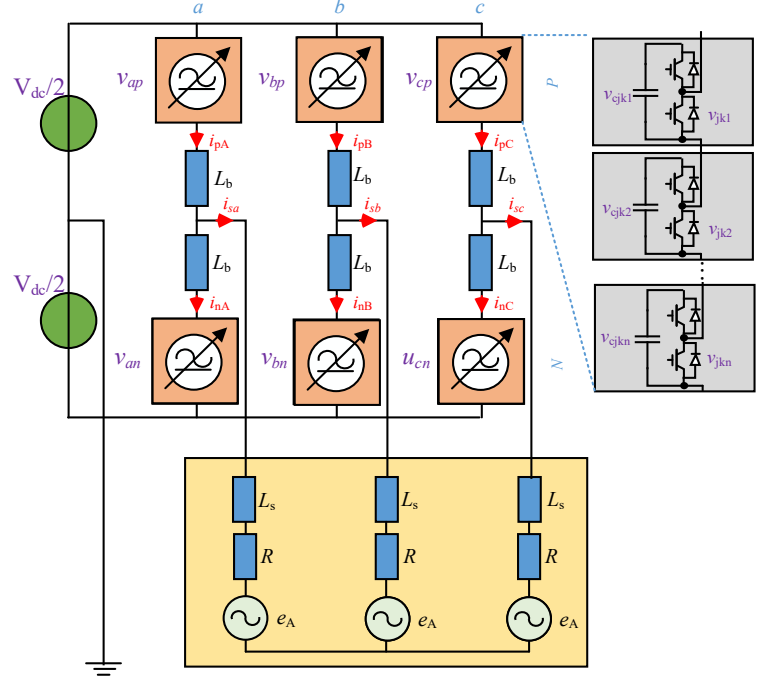

Figure 1. The simplified circuit of MMC-ESSC

Under ideal working conditions, the voltage and current on the AC side, the capacitor voltage of the bridge arm submodule is input, and the dynamic relationship of the bridge arm current is:

$$
\left\{\begin{array}{l}
\frac{V_{\mathrm{dc}}}{2}-v_{j p}-e_{j}=L_{\mathrm{b}} \frac{d i_{j p}}{d t}+R i_{s j}+L_{\mathrm{s}} \frac{d i_{s j}}{d t} \\
-\frac{V_{\mathrm{dc}}}{2}+v_{j n}-e_{j}=-L_{\mathrm{b}} \frac{d i_{j n}}{d t}+R i_{s j}+L_{\mathrm{s}} \frac{d i_{s j}}{d t}
\end{array}\right.
$$

The bridge arm currents have the following relationship with the AC side current, DC bus current, and phase-to-phase circulating current:

$$
\left\{\begin{array}{l}
i_{j p}=\frac{i_{\mathrm{dc}}}{3}-\frac{i_{\mathrm{s} j}}{2}+i_{\mathrm{zj}} \\
i_{j n}=\frac{i_{\mathrm{dc}}}{3}+\frac{i_{\mathrm{s} j}}{2}+i_{\mathrm{zj}}
\end{array}\right.
$$

Combining formula (1) and formula (2), the relationship between the switching state of the submodule and the output variable on the grid side can be obtained:

$e_{j}+\frac{\sum_{i=1}^{N} S_{j p} \times v_{c j p i}-\sum_{i=1}^{N} S_{j n} \times v_{c j n i}}{2}=\left(L_{\mathrm{s}}+\frac{L_{\mathrm{b}}}{2}\right) \frac{d i_{\mathrm{s} j}}{d t}+R i_{\mathrm{s} j}$

\section{Constraint Satisfied Model Predictive Control Strategy for MMC Energy Storage System Based on Super Capacitor}

Model predictive control is a kind of modern control theory. By predicting the future state of the power electronic system, the optimal switching state is screened and applied to the system to obtain excellent control effects. The basic control principle is shown in the figure 2.

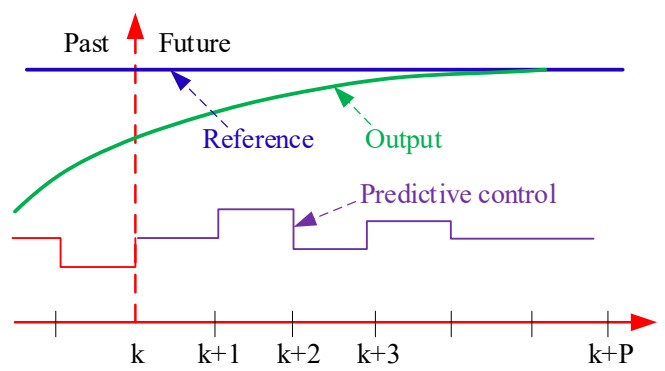

Figure 2. Basic principle diagram of model predictive control

\subsection{Discrete domain mathematical model establishment}

The derivative term can be discretized by employing the Forward Euler approximation

$$
\frac{\mathrm{d} x}{\mathrm{~d} t}=\frac{x(k)-x(k-1)}{T_{s}}
$$

In the above, the relationship between the output variable of the power grid and the switching state of the sub-module and the state of the internal sub-module has been obtained, and the above formula is discretized to obtain the prediction model of the system, as shown in formula (5). To simplify the following equation, $K_{1}=\left(L_{\mathrm{s}}+L_{\mathrm{b}} / 2\right) / T_{\mathrm{s}}$ and $K_{2}=K_{1}+\mathrm{R}$.

$i_{s j}(\mathrm{k}+1)=\frac{1}{K_{2}}\left(\frac{\sum_{i=1}^{N} S_{j n}(\mathrm{k}+1) \times v_{c i n i}(\mathrm{k}+1)-\sum_{i=1}^{N} S_{j p}(\mathrm{k}+1) \times v_{c j p i}(\mathrm{k}+1)}{2}-e_{j}(\mathrm{k}+1)-K_{1} i_{s j}(\mathrm{k})\right)$

Since the pressure equalization of the energy storage system is good and stable, the above formula can be simplified to the following formula:

$$
i_{\mathrm{sj}}(\mathrm{k}+1)=\frac{1}{K_{2}}\left(\frac{S_{j n}(\mathrm{k}+1) \times V_{c}^{a v g}-S_{j p}(\mathrm{k}+1) \times V_{c}^{a v g}}{2}-e_{j}(\mathrm{k})-K_{1} i_{\mathrm{sj}}(\mathrm{k})\right)
$$

The instantaneous predicted value of the capacitor voltage of the future submodule can be predicted:

$$
v_{\mathrm{c} j k n}(\mathrm{k}+1)=v_{\mathrm{c} j k n}(\mathrm{k}+1)+S_{j k n} \frac{T_{\mathrm{s}}}{\mathrm{C}} i_{j k}(\mathrm{k})
$$

The instantaneous predicted value of bridge arm circulation can be expressed as:

$$
i_{\mathrm{zj}}(\mathrm{k}+1)=\frac{T_{\mathrm{s}}}{2 L_{\mathrm{b}}}\left(V_{\mathrm{dc}}-v_{j p}(\mathrm{k}+1)-v_{j n}(\mathrm{k}+1)\right)+i_{z j}(\mathrm{k})
$$

In order to eliminate the delay caused by the model predictive control, delay compensation is performed 
during the prediction process, and the corresponding output current prediction value can be expressed as

$$
i_{\mathrm{s} j}(\mathrm{k}+2)=\frac{1}{K_{2}}\left(\frac{v_{j n}(\mathrm{k}+2)-v_{j p}(\mathrm{k}+2)}{2}-e_{j}(\mathrm{k}+1)-K_{1} i_{s j}(\mathrm{k}+1)\right)
$$

\subsection{Objective function establishment}

In order for the controlled object to approach the given value to the greatest possible extent, it is necessary to select the optimal output states and corresponding submodule switching schemes. In the MMC-ESSC system, the main control targets are: active and reactive power tracking given, sub-module capacitor voltage balance, phase-to-phase circulating current suppression and submodule switching frequency. By adjusting the weighting factors in different control targets, multiple control targets are normalized and converted into a single online optimization solution problem. Establish the following objective equation:

$J_{k \min }=\lambda_{1}\left|i_{s k}^{*}-i_{s k}(k+1)\right|+\lambda_{2} \sum_{n}\left|v_{c j k n}^{*}-v_{c j k n}(k+1)\right|+\lambda_{3}\left|i_{z k}^{*}-i_{z k}(k+1)\right|+\lambda_{4}\left|f_{k}^{*}-f_{k}(k+1)\right|$
The established objective equation $J_{\min }$ contains four objective terms. By adjusting the weight factors $\lambda_{\mathrm{p}}(\mathrm{p}=1,2,3,4)$, the multi-objective control is transformed into a function solving problem. The function $J_{\min }$ value is the smallest, it proves that the corresponding sub-module switching state is closest to the control targets.

\subsection{Rolling optimization process}

In order for the controlled object to approach the given value to the greatest possible extent, it is necessary to select the optimal output state and its corresponding submodule switching scheme. In the MMC-HVDC system, the main control targets are: active and reactive power tracking given, sub-module capacitor voltage balance, phase-to-phase circulating current suppression. By adjusting the weighting factors in different control targets, multiple control targets are normalized and converted into a single online optimization solution problem. Establish the following objective equation:

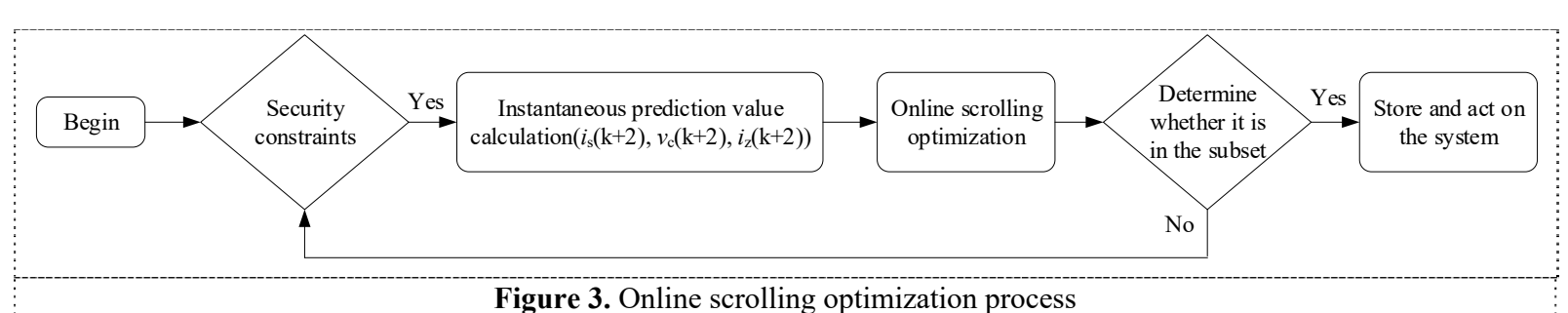

Figure 3. Online scrolling optimization process

\section{Experimental results of constraint satisfaction model predictive control for MMC-ESSC}

To further verify the correctness of the CS-MPC strategy for MMC-ESSC, a MMC-ESSC experimental platform each phase unit contains 8 sub-modules in series was built, and the correctness of the above control strategy was initially verified.

\subsection{Analysis of constraint satisfaction model predictive control steady state experiment results}

The steady-state experimental waveform of MMCESSC system based on constraint satisfaction model predictive control is shown in Figure 4. The figure respectively shows the voltage equalization of the submodule supercapacitor and the three-phase output voltage waveform. It can be seen from the experimental results that the supercapacitors in each sub-module have a good voltage stabilization condition and achieve energy balance. By setting appropriate weighting factors, the switching frequency of sub-modules can be effectively reduced, the switching loss of the system can be reduced, and the conversion efficiency of the system can be improved. 


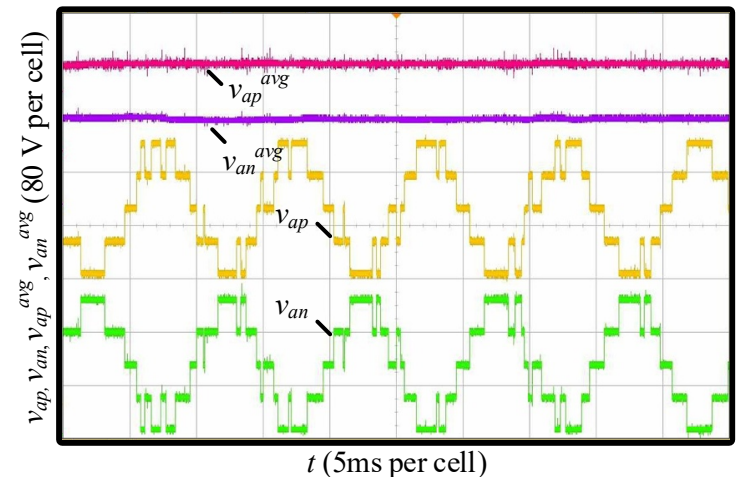

(a) Average value of upper and lower bridge arm super capacitors

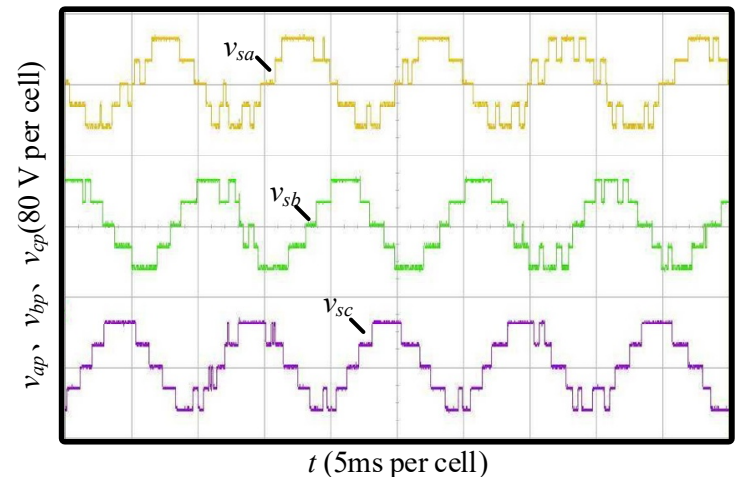

(b) Output phase voltage waveforms of MMC-ESSC

Figure 4. Steady-state experimental results under reduced switching frequency

The system output phase voltage current and line voltage waveforms are shown in Figure 5, and the output currents are set to 10A. The analysis of the experimental results shows that safety constraints are added to the model predictive control strategy, and the output line voltage and phase voltage have no cross-level jumps. On the premise of ensuring the output quality of the system, avoid local temperature rise caused by short-term voltage jump and improve the safe operation ability of the system.

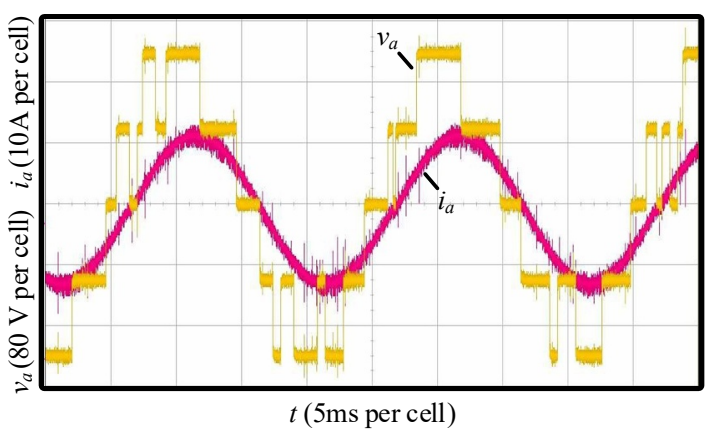

(a) Output phase voltage and current waveforms of MMC-ESSC

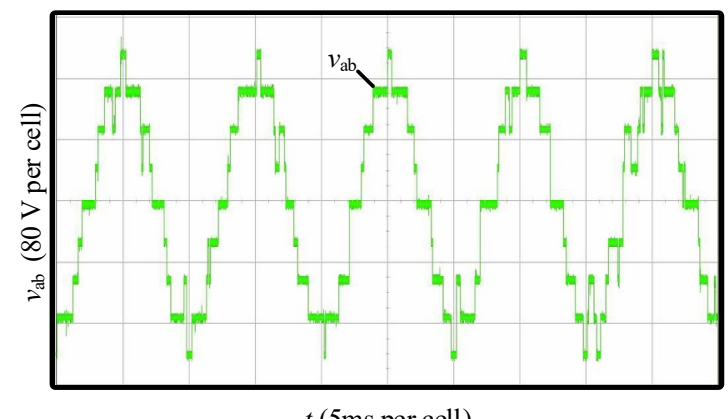

(b) Output line voltage waveform of MMC-ESSC

Figure 5. Steady-state experimental results under safety constraints

\subsection{Analysis of constraint satisfaction model predictive control dynamic state experiment results}

In the initial stage, the system gives a current command as $8 \mathrm{~A}$, and then the command changes to $15 \mathrm{~A}$. The MMC-ESSC system based on constraint satisfaction model predictive control can quickly predict the states of the next control cycle and start online optimization. The proposed control strategy can find the optimal switching states of the sub-modules and act on the energy storage system. As shown in the figure 6, within $1 \mathrm{~ms}$, the setpoint can be quickly tracked, and there is no oscillation and overshoot in the dynamic response process. The experimental results show that the proposed control strategy has excellent dynamic response characteristics.
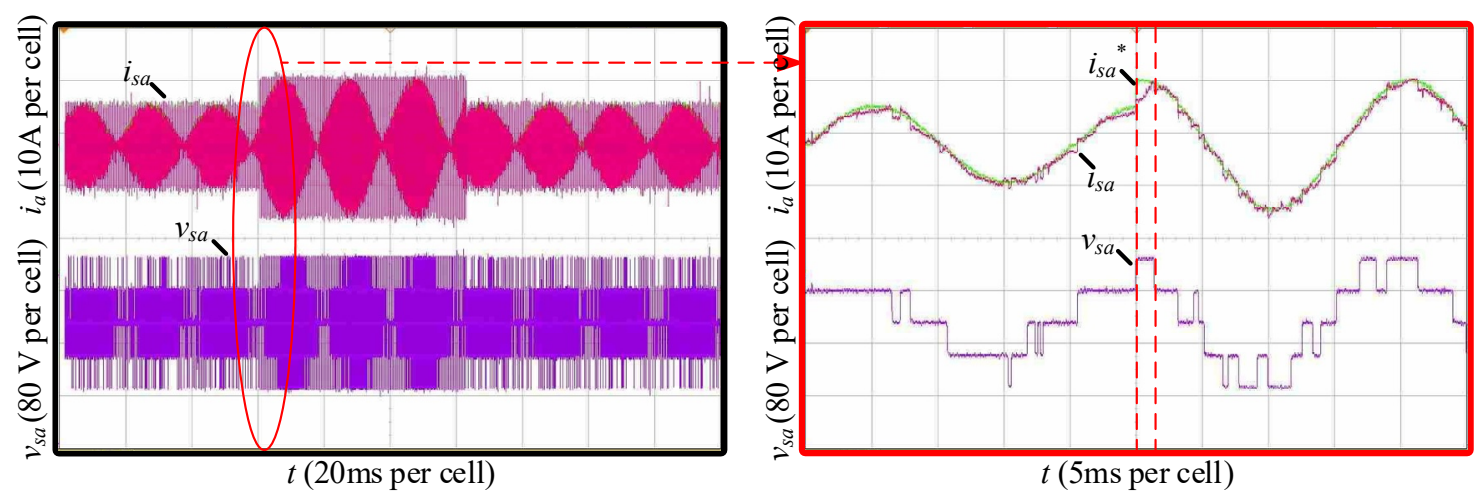

Figure 6. MMC-ESSC dynamic response experiment results 


\section{Conclusion}

This paper proposes a constraint satisfaction model predictive control method for MMC energy storage system based on super capacitor. In the article, the operation mechanism of MMC energy storage system is analyzed, and the discrete domain mathematical model of MMC-ESSC is established. The article studies the prediction method of the future internal and external variables of the system, the rolling optimization mechanism and the method of establishing the objective function, and finally carries on the experiments verification. The analysis of experimental results shows that proposed control technology has high dynamic characteristics and efficiency. A new idea is proposed for the research on the control strategy of future MMC energy storage systems.

\section{Acknowledgment}

This work was supported by the State Grid Corporation Headquarters Science and Technology Project, i.e., Research on Fault Detection and Ride-through Technology of DC Distribution Network without DC Circuit Breaker.

\section{References}

1. Flourentzou N, Agelidis V G, Demetriades G D. VSC-based ESSC power transmission systems: an overview $[\mathrm{J}]$. Power Electronics, IEEE Transactions on, 2009, 24(3): 592-602.

2. ZHAO Xin, ZHAO Chengyong, LI Guangkai, et al. Submodule Capacitance Voltage Balancing of Modular Multilevel Converter Based on Carrier Phase Shifted SPWM Technique[J]. Proceeding of the CSEE, 2011, 31(21): 48-55.

3. Venkat A N , Hiskens I A, Rawlings J B , et al. Distributed MPC Strategies With Application to Power System Automatic Generation Control[J]. IEEE Transactions on Control Systems Technology, 2008, 16(6):1192-1206.

4. Liu, Wenxia, Yahui. Reliability model of MMCbased flexible interconnection switch considering the effect of loading state uncertainty[J]. IET Power Electronics, 2019.

5. Ruoping L, Yinhong L I, Hao X, et al. Research on adaptability of DC pole protection to fault occurred during start-up process for MMC-HVDC[J]. Electric Power Automation Equipment, 2019.

6. Fei Rong, Jiajun Yan, Wenlong Sun, et al. Control strategy of wind energy conversion system based on $\mathrm{H}-\mathrm{MMC}$ under asymmetrical grid faults[J]. Power Electronics, IET, 2019. 